\title{
Microfluidic Nozzles for Additive Manufacturing of Gels and Soft Materials
}

\author{
P. Ravi Selvaganapathy \\ McMaster University, Canada \\ Canada
}

3D printing and additive manufacturing technologies have been developed over the past 30 years and have become mainstream in the past 10 years. Extrusion based 3D printing is the technique that has the most widespread use and is suitable for use with thermoplastic polymers. However, it cannot be easily used for multimaterial combinations or used with reactive or crosslinking polymers such as gels or soft materials such as silicones. In this talk, I will present an overview of bioprinting technologies, identify that the microfluidic nozzle design is a crucial element to customize it for printing gels, extracellular matrices (ECMs) and soft materials as well as discuss some of the innovative designs that have been developed recently. These emerging nozzle designs will enable precise positioning of cells, ECMs and soft materials as well as integrate vascular channels into them enabling the application of such constructs in the area of drug discovery, tissue engineering and building artificial organs. 